\title{
Computer-aided detection/diagnosis of breast cancer in mammography and ultrasound: a review.
}

\begin{abstract}
Breast cancer is the most common form of cancer among women worldwide. Early detection of breast cancer can increase treatment options and patients' survivability. Mammography is the gold standard for breast imaging and cancer detection. However, due to some limitations of this modality such as low sensitivity especially in dense breasts, other modalities like ultrasound and magnetic resonance imaging are often suggested to achieve additional information. Recently, computer-aided detection or diagnosis (CAD) systems have been developed to help radiologists in order to increase diagnosis accuracy. Generally, a CAD system consists of four stages: (a) preprocessing, (b) segmentation of regions of interest, (c) feature extraction and selection, and finally (d) classification. This paper presents the approaches which are applied to develop CAD systems on mammography and ultrasound images. The performance evaluation metrics of CAD systems are also reviewed.
\end{abstract}

Keyword: Computer-aided detection; Computer-aided diagnosis; Breast cancer; Mammography; Ultrasound 